\title{
The Contemporaneous and Impending Developments for Combatting COVID-19
}

\author{
Singh $\mathrm{NK}^{1 *}$ and Kuldeep $\mathbf{M}^{2}$ \\ ${ }^{1}$ Department of Electrical and Computer Engineering, University of California, USA \\ ${ }^{2}$ Department of Nano Engineering, University of California, USA
}

*Corresponding author: Naveen K Singh, Department of Electrical and Computer Engineering, University of California-San Diego, La Jolla, CA 92093, USA, Email: nsingh@ucsd.edu

\section{Editorial}

Volume 4 Special Issue 1

Received Date: October 29, 2020

Published Date: November 17, 2020

DOI: $10.23880 /$ jidtm-16000S1-008

\section{Abstract}

Severe Acute Respiratory Syndrome Coronavirus 2 (SARS-CoV-2), the causative viral agent for the COVID-19 infection has rapidly spread worldwide. The SARS-CoV-2 infection spread around the globe ( 198 countries) and true statistics of this pandemic are difficult to estimate due to significantly limited healthcare capability around the world. For the alleviation, the effects of the SARS CoV-2 virus on public health and the economy, a vaccine is urgently needed. Currently, more than 10 vaccine candidates have reached the final phase of the clinical trial. The global scale-up of vaccine trials requires a massive testing and immune monitoring effort that is unprecedented in modern science. While there are many vaccination strategies, the common theme is that these must elicit sustained immunity that is predicated on the production of neutralizing or blocking antibodies that recognize exposed protein epitopes. Today this process remains entirely laboratory bound, is costly, and in the availability of common assay for assessing immunity across multiple vaccines hence an urgent preparedness needed to overcome forthcoming challenges.

Keywords: SARS-CoV-2; Swine; Influenza; Zika; Ebola

The outbreak of SARS-CoV-2 from Wuhan, China in late 2019 has now become pandemic, which has tolled 5 million death counts till date [1]. With the increasing number of cases globally, this pathogenic virus has already joined the past pandemics impacted by Avian and H1N1 (swine) influenza, Zika, Ebola, human immunodeficiency virus, Middle East respiratory syndrome Coronavirus, and severe acute respiratory syndrome. This virus-borne outbreak has shaped the instances as pandemic due to the human contacts led by certain essential activities such as crossborder immigrations, globalization, societal unrest, etc. Not only these, but the environmental factors have also enhanced the transmission of causative entity rapidly across the globe [2]. This pandemic not only impacted the healthcare services but also it has greatly impacted commercial activities at almost every level leading to the global crisis. For combatting these crises, several efforts have constantly been given everywhere to revive the normal situations, where research and development on the rapid screening, confirmatory diagnostics, and vaccine have been on the utmost priority [3]. In this context, academic institutions as well as healthcare giants have partnered to deliver accurate and authentic diagnostic tools for the rapid screening and confirmatory tests of SARS-CoV-2 infections [4]. The technology-empowered solutions have great support for the management of the COVID-19 emergencies by introducing the rapid screening AI-powered models and thermal sensors [5]. Advancements in technologies have also leveraged various equipment's for 
assisting patient management in severe conditions. Apart from these first lines of preparedness, the development of confirmatory diagnostic kits has also played a key role in public health management in the crisis. These include the RT-qPCR and the antigen-based portable diagnostics kits.

Another form of preparedness / fight against any pandemic diseases is immunization, which is commonly done by vaccination6. The race for developing an effective SARS-CoV-2 vaccine is continuing at full pace and thus there are extensively collaborated efforts that have been given by academia and healthcare industries [6,7]. Nevertheless, the milestones in this context are quite a few including the vaccines developed by Moderna, AstraZeneca, J\&J (Jansen), Novarx, Sanofi GSK, Merck/AVI, Pfizer/NTECK targeting selective epitopes present on the viral capsid and the genetic materials. Although, their neutralizing capabilities are decently higher in the laboratory trials; these are still in the clinical trial and thus the entire globe is extremely hopeful [7]. While current vaccine approaches vary in delivery mechanism (e.g., purified protein, non-replicating viral vectors, or inactivated SARS-CoV-2 virus, the end goal is to induce the immune system to produce neutralizing antibodies directed to coronavirus antigen(s). Recent work by Rogers and others have shown potent neutralizing activity associated with key regions of the spike glycoprotein S present at the capsid of the virus [8]. Many of these are associated with the receptor-binding domain, which promotes the first step of viral entry by binding to exposed ACE2 receptors in the respiratory tract lining. As conclusively, significant numbers of vaccine candidates have selected protein $\mathrm{S}$ regions for which blocking antibodies might inhibit viral entry. These vaccines are then monitored for their ability to produce sustained immunologic responses directed to these target domains. The vaccine response or immunization status has been confirmed by quantitative detection of antibodies (antibody titer) in usual cases upon the immunization. In COVID-19, the antibody developed against the vaccines has been titrated with specific SARS CoV-2 epitopic domain. As per normal immune response after getting exposed to the SARS CoV-2 virus, specific antibodies directed towards viral-specific antigens may be detected within a week of vaccination or symptoms (IgM as early as day 7 and IgG $>14$ days post-infection) [9]. These antibodies are typically measured in blood using enzyme immunofluorescence assays that require expertly trained personnel and expensive laboratory equipment [10]. The rapid tests such as the Gene Xpert, Euroimmun, and Healgen are commercially available for their validation. However, each vaccine trial requires adapted test development and careful validation [11]. Many of these efforts are employing mobile clinics and taking place in over 30 countries [7], yet the determination of immunity is still empirical and technologies for measurement remain laboratory-bound, time-consuming, and costly. This presents a potential bottleneck in vaccine trial logistics, and muchheralded immunity-checks and skilled manpower. There is a critical need to develop simple, scalable methods to track response to vaccines and ultimately determine levels of SARS-CoV-2 immunity outside of laboratory settings. Not only this, the major challenge at the post vaccination stage would be expected for confirming the immunization status of the immunized individuals; where the numbers would be in thousands, sometimes tens of thousands. The preparedness in this phase would be extremely crucial and its success will rely on the availability of the reliable point of care rapid testing kits in large scale for avoiding pre- and post-vaccination surge of the covid cases.

\section{References}

1. United States Coronavirus: 6,718,969 Cases and 198, 643 Deaths-Worldometer.

2. Morens DM, Fauci AS (2020) Emerging Pandemic Diseases How We Got to COVID-19. Cell 182(5): 10771092

3. Pascarella G, Alessandro Strumia, Chiara Piliego, Federica Bruno, Romualdo Del Buono, et al. (2020) COVID-19 diagnosis and management a comprehensive review J Intern Med 288(2): 192-206.

4. Singh NK, Partha Ray, Aaron F Carlin, Celestine Magallanes, Sydney C Morgan, et al. (2020) Hitting the diagnostic sweet spot: Point-of-care SARS-CoV-2 salivary antigen testing with an off-the-shelf glucometer.

5. Budd J, Benjamin S Miller, Erin M Manning, Vasileios Lampos, Mengdie Zhuang, et al. (2020) Digital technologies in the public-health response to COVID-19 Nat Med 26(8): 1183-1192.

6. Jeyanathan M, Sam Afkhami, Erin M Manning, Vasileios Lampos, Mengdie Zhuang, et al. (2020) Immunological considerations for COVID-19 vaccine strategies Nat Rev Immunol 20(10): 615-632

7. Krammer F (2020) SARS-CoV-2 vaccines in development. Nature 586: 516-527.

8. Rogers TF, Fangzhu Zhao, Deli Huang, Nathan Beutler, Alison Burns, et al. (2020) Isolation of potent SARS-CoV-2 neutralizing antibodies and protection from disease in a small animal model. Science 369(6506): 956-963.

9. Centers for Disease Control and Prevention CDC (2019) Information for Laboratories about Coronavirus (COVID-19). 
10. Wang C, Wentao Li, Dubravka Drabek, Nisreen M A Okba, Rien van Haperen, et al. (2020) A human monoclonal antibody blocking SARS-CoV-2 infection Nat Commun11(1): 2251
11. Kubina R, Dziedzic A (2020) Molecular and Serological Tests for COVID-19 A Comparative Review of SARS-CoV-2 Coronavirus Laboratory and Point-of-Care Diagnostics 10(6): 434. 